\title{
LA APLICACIÓN DE LOS CONCEPTOS DE GÉNERO, MACROESTRUCTURA Y CONVENCIONES TEXTUALES A LA TRADUCCIÓN DE TESTAMENTOS FRANCESES AL ESPAÑOL Tanagua Barceló Martinez. \\ Universidad de Málaga
}

\section{ABSTRACT}

In translation studies research conducted during the last decades, there are certain key concepts which have been subject of numerous studies, discussions and analyses. These studies usually focus on the definition, naming conventions and limits of these key concepts. Some of these discussions are still the core theme of current researches and terminological confusion still prevails. This paper deals with some of these key concepts, namely, text genres and text macrostructure. They have been grouped and applied to the translation of a specific type of text: the French testament authentique. Our goal is to show the importance of the pre-translation phase in this text type, while trying to stay away from the debate by different currents and existing stances, although some of them will be used to set the theoretical foundations of this paper.

KEYWORDS: text genre, macrostructure, text conventions, languages for specific purposes, legal language, wills.

\section{RESUMEN}

En los estudios traductológicos realizados a lo largo de las últimas décadas, algunos conceptos han sido objeto de numerosos estudios, discusiones y análisis que se centran, principalmente, en las definiciones de dichos conceptos, en su denominación y en el establecimiento de sus límites. Algunas de esas discusiones continúan aún siendo el eje de diferentes investigaciones y el confusionismo terminológico sigue reinando. Entre los mencionados conceptos queremos resaltar los de géneros textuales y macroestructura textual que, en el presente artículo, hemos agrupado y aplicado a la traducción de un tipo de documento concreto: el testamento abierto francés. Nuestro objetivo es mostrar la importancia que tienen en la fase de pretraducción, intentando mantenernos al margen de las discusiones entre las diferentes corrientes y posturas existentes, a pesar de tener que recurrir a ellas para sentar las bases teóricas de nuestro artículo. 
PALABRAS CLAVE: género textual, macroestructura, convenciones textuales, lenguas de especialidad, lenguaje jurídico, testamento.

\section{INTRODUCCIÓN}

Contrariamente a lo que muchos imaginan, traducir es una tarea harto complicada que exige, por parte del que la acomete, el manejo de toda una serie de destrezas y aptitudes, así como el conocimiento de diversos y variados aspectos relacionados con la traductología, la lingüística y, por supuesto, con el contenido de los textos a los que el traductor se enfrenta. En ese sentido, Cristina Valderrey (2006: 68), parafraseando a Gémar, afirma que

"Para trasladar un texto especializado es necesario conocer (comprender y saber utilizar) el lenguaje propio de la especialidad. Y ello significa dominar tanto el léxico como el discurso utilizado por los expertos del campo, es decir, la manera en la que se dicen las cosas en esa especialidad."

La acción de traducir debe contener más fases además de la estrictamente ceñida al traspaso del contenido de un texto en una lengua dada a otra lengua. A la hora de enfrentarse a la traducción de cualquier texto, el traductor deberá adoptar una estrategia traslativa y seguir un determinado modelo que le permita analizar el texto en cuestión desde diferentes puntos de vista atendiendo a parámetros vinculados a diversos aspectos que variarán en mayor o menor medida en función de la finalidad de la traducción y del contexto en el que el texto se produzca.

Valderrey (2006: 59-91) describe de forma profusa un modelo de análisis textual con vistas a la traducción en el que hace especial hincapié en los géneros textuales y en la importancia de la estructura y del ordenamiento de la información en los textos de origen jurídico. Atendiendo en parte al mencionado modelo, vamos a centrarnos en un tipo de documento jurídico concreto, el testamento abierto francés, e intentaremos analizar hasta qué punto el estudio, conocimiento, análisis y manejo de los géneros, de la macroestructura y de las convenciones textuales y ortotipográficas ayudan de cara a la traducción profesional de dicho tipo de documento. Para ello, deberemos considerar diferentes conceptos, algunos de los cuales, a pesar de haber sido estudiados hasta la fecha de forma exhaustiva por diferentes autores, no gozan de unanimidad de criterios en lo que a su definición y sus límites se refiere por un lado y su aplicación en la enseñanza o la práctica de la traducción por otro. 
Para conformar el concepto global que para nosotros abarca el término género textual y, más concretamente, el género jurídico, nos hemos basado en las definiciones de tres autoras de prestigio del panorama investigador actual. Según Valderrey (2006: 71):

"los géneros son formas discursivas estereotipadas, de carácter social y cultural, que se manifiestan como recursos comunicativos efectivos para solucionar tareas comunicativas específicas; se definen principalmente por sus características externas, al poseer particularidades estructurales y de formulación características. Son, por tanto, producciones prototípicas y, en ese sentido, sistematizables en su estudio. ${ }^{1}$

Isabel García Izquierdo (2007: 122) define género como la "expresión tangible de las convenciones textuales y contextuales contenidas en los textos representativos de determinados ámbitos socio-profesionales." Para dicha autora (Izquierdo: 2007: 122), cualquier forma textual que responda a determinadas convenciones y que esté determinada por factores culturales independientemente del ámbito al que pertenezca (especializado o no) puede ser considerado un género.

Por su parte, Anabel Borja² (2007a: 144) habla de género jurídico en los siguientes términos:

"[...] existen unos modelos rígidos y repetitivos que impone la cultura jurídica heredada, los hábitos profesionales, las costumbres sociales e incluso la formación académica. Todo ello conforma una selección de contenidos, una manera de exponer los hechos, una retórica y una percepción del mundo que aparecen plasmados de forma inequívoca en cada realización textual del ámbito jurídico. Si a esta realización textual concreta le

1 Esta misma autora (Valderrey 2006: 71) defiende además tres ideas que nos parecen especialmente interesantes. Así, afirma que "los géneros consisten en conjuntos limitados de ejemplares textuales con rasgos específicos en común; son reconocidos por los hablantes al compartir tanto el formato externo como los parámetros situacionales de producción" y que "se trata además de una noción delimitadora que permite agrupar bajo una misma categoría conjuntos de actualizaciones textuales particulares". Por último, añade que "en su identificación se integran tanto parámetros externos (situacionales, socioculturales, etc.) como internos de distinta naturaleza (estructurales, sintácticos, etc.)."

2 La autora (Borja 2007a: 141-153) hace un recorrido por la evolución de la teoría general de los géneros y defiende la pertinencia de la aplicación de este concepto en el proceso traslativo. Asimismo, ofrece un panorama actual de los estudios realizados que versan sobre la aplicación más o menos directa del concepto de género a los textos jurídicos. 
sumamos un objetivo comunicativo claro o una función jurídica específica, el resultado es el concepto de género jurídico."

En este sentido, el testamento se presenta como un género jurídico independiente que responde a esta definición. No obstante, creemos que existe un escalón superior y otro inferior a los cuales el traductor debería recurrir a la hora de contextualizar un testamento.

En un nivel superior, habremos de ubicar el testamento dentro del ordenamiento jurídico. Los ordenamientos jurídicos francés y español presentan una división en ramas del Derecho muy parecida y, dentro del denominado Derecho Nacional o Interno, ambos distinguen entre Derecho Público y Derecho Privado. En España, el Derecho Público comprende el Derecho Penal, el Laboral, el Financiero y el Tributario. Por su parte, el Derecho Privado abarca el Derecho Civil y el Mercantil. El Derecho Civil se subdivide, a su vez, en diferentes ramas: Derecho de la Personas, Derecho de las Obligaciones, Derecho de las Cosas o Bienes, Derecho de Familia y Derecho de Sucesiones. Dentro de este último, el testamento sería uno de los documentos producidos más característicos ${ }^{3}$.

En un nivel inferior, deberemos discernir cuáles son los diferentes tipos de testamentos existentes, y ello con el objeto de establecer las similitudes y las diferencias existentes entre las culturas jurídicas de los países aquí involucrados. Según el Código Civil español, podemos distinguir entre testamentos comunes y testamentos especiales. Dentro del primer grupo se incluyen el testamento ológrafo, el abierto y el cerrado. Forman parte de los testamentos especiales el militar, el marítimo y el hecho en país extranjero. El ordenamiento jurídico francés distingue entre testaments ordinaires y testaments privilégiés. Dentro del primer grupo se incluyen el testament olographe, el authentique y el mystique. Forman parte del segundo grupo el testament militaire, el testament fait sur mer y el testament fait en pays étranger. Dentro de este nivel de análisis y caracterización, podríamos afirmar que el testamento abierto y el testament authentique son equivalentes ${ }^{4}$.

Aquí hemos utilizado los conceptos de género, tal y como aparece definido más arriba, para referirnos al testamento en general en el sentido en el que le podemos atribuir las características ya mencionadas y el de tipo para

\footnotetext{
${ }^{3}$ En Francia, el Droit des successions es una subdivisión del Droit civil, que, a su vez, pertenece al Droit privé, tal y como hemos visto que ocurre en el seno del ordenamiento jurídico español.

${ }_{4}^{4}$ Debemos matizar que las clasificaciones presentadas responden a criterios de forma de los testamentos. Cada uno de los tipos mencionados exige una serie de requisitos legales de obligado cumplimiento para que el documento tenga valor jurídico. Estos requisitos, que no son exactamente los mismos para cada uno de los dos ordenamientos jurídicos, dan lugar a la existencia y la presencia de fórmulas fraseológicas que, de algún modo, caracterizan cada una de las partes de que debe constar un tipo de testamento dado. La traducción de dicho contenido y el establecimiento de las equivalencias pertinentes no son tan automáticos como la comparación formal que acabamos de establecer.
} 
remitirnos a los diferentes modos de testamento existentes. Todos ellos se enmarcarían dentro del denominado lenguaje jurídico que, a su vez, se inscribe dentro de las lenguas de especialidad ${ }^{5}$. Este primer paso podría calificarse como la fase de ubicación del texto atendiendo a su procedencia (jurídica en este caso) y a las diferentes formas que puede presentar (tipos de testamentos). Queremos subrayar la pertinencia de este tipo de análisis. A pesar de ser más productivo en unos casos que en otros, contribuye siempre a ubicar el texto que debemos traducir. En el caso de los testamentos, y debido a que éstos responden en gran medida a la definición de documento-tipo, este proceder resulta especialmente esclarecedor y puede desembocar en estudios que sistematicen las dificultades propias de un determinado género textual ${ }^{6}$. En este punto, y tras llevar a cabo esa primera fase de aproximación, el testamento abierto francés ha pasado de ser "sólo" un texto a ser un documento procedente del ámbito jurídico, concretamente un documento emanante de la aplicación del Derecho de Sucesiones y responde a una de las formas de testamento recogidas por el Código Civil francés.

\section{LA MACROESTRUCTURA TEXTUAL}

Actualmente, tampoco existe un consenso generalizado en cuanto a la definición de macroestructura textual. Nosotros entendemos dicho concepto en el sentido de Valderrey (2006: 80), quien afirma que macroestructura es la organización prototípica del texto, y añade que

"representa también la estructura conceptual del texto, pues las distintas partes actúan como elementos identificadores de contenido y permiten la obtención de información semántica general o, en otras palabras, una comprensión global del texto".

$\mathrm{Al}$ aplicar este concepto al ámbito del derecho, la autora defiende que

"los textos jurídicos se caracterizan [...] por la naturaleza restrictiva de sus estructuras y por la sistematicidad en la presentación de la información." "Conviene redundar en la importancia de la distribución del contenido en el texto jurídico. Dicha distribución está sometida a una organización preestablecida que se sirve, entre otros recursos, de las fórmulas

\footnotetext{
${ }^{5}$ La definición, la denominación y el establecimiento de los límites de las lenguas de especialidad es otro de los aspectos que mayores discusiones ha suscitado y sigue suscitando en la investigación traductológica.

${ }^{6}$ En este sentido, Anabel Borja (2007a: 153) afirma que "Las tipologias textuales dentro de un lenguaje de especialidad facilitan la sistematización de la traducción mediante el análisis de corpora de documentos tipo."
} 
estereotipadas. Estas formas convencionales cumplen una función demarcativa fundamental al contribuir a delimitar las partes en que se estructura cada escrito... ${ }^{\prime \prime}$

En efecto, determinados tipos de textos obedecen más que otros a ese ordenamiento preestablecido de la información que, sin duda, ayuda a caracterizar un texto y a establecer paralelismos con textos similares (textos paralelos) en otras lenguas. Los textos jurídicos, por lo general, se caracterizan por ser muy formales y por la ordenación sistemática de su contenido, aunque este aspecto no es exclusivo de este ámbito ${ }^{8}$. El testamento abierto obedece a esta característica y se incluye entre aquellos documentos cuyo contenido se encuentra perfectamente dividido en diferentes partes que conforman su macroestructura. Y esto tanto en los testamentos abiertos franceses como en los españoles.

El contenido de dichas partes se refiere al desarrollo lógico del acto de otorgamiento de un testamento abierto por un lado y, por otro, es el fiel reflejo del cumplimiento de las formalidades y exigencias impuestas por las diferentes normativas en lo que a la realización de este tipo de actos se refiere.

Presentamos a continuación una tabla comparativa de las macroestructuras del testamento abierto y del testament authentique 9 :

\begin{tabular}{|l|l|}
\hline $\begin{array}{l}\text { MACROESTRUCTURA } \\
\text { TESTAMENTO ABIERTO } \\
\text { (ESPANA) } 10\end{array}$ & $\begin{array}{l}\text { MACROESTRUCTURA } \\
\text { TESTAMENT AUTHENTIQUE } \\
\text { (FRANCIA) }\end{array}$ \\
\hline Número de protocolo & Número de protocolo \\
\hline $\begin{array}{l}\text { Lugar, fecha y hora del } \\
\text { otorgamiento }\end{array}$ & Fecha y lugar del otorgamiento \\
\hline $\begin{array}{l}\text { Nombre, colegio de pertenencia del } \\
\text { notario fedatario }\end{array}$ & Nombre del notario fedatario \\
\hline
\end{tabular}

${ }^{7}$ Hay autores que interpretan el concepto en otro sentido. Es el caso de Van Dijk (Dijk: 1980, 52-57), para quien el texto es una unidad de comunicación que organiza su contenido en el plano global en dos tipos de estructuras: la macroestructura y la superestructura textuales. La macroestructura textual da cuenta del contenido semántico que representa el tema del texto. En cambio, la superestructura textual representa el esquema de composición del texto, esto es, la forma como el contenido se organiza en partes reconocibles." Según Van Dijk (Dijk: 1980: 53), "una superestructura puede caracterizarse intuitivamente como la forma global de un discurso y las relaciones (jerárquicas) de sus respectivos fragmentos."

8 Ténganse en cuenta, por ejemplo, las recetas de cocina o los prospectos de medicamentos.

9 Testament authentique es el nombre que recibe en francés el documento que en España se conoce como testamento abierto. No obstante, queremos subrayar la necesidad que el traductor tiene de conocer tanto las denominaciones (es decir, el aspecto terminológico) como el contenido que abarcan, con el objeto de establecer las equivalencias pertinentes y de saber cuáles son los vacíos conceptuales existentes.

10 Según la propuesta de Amparo Hurtado Albir (1999: 165). 
ENTREC ULTURAS Número 1. ISSN: 1989-5097. Fecha de publicación: 27-03-2009

\begin{tabular}{|l|l|}
\hline Datos personales del testador & Tipo de testamento \\
\hline $\begin{array}{l}\text { Reconocimiento del notario de la } \\
\text { capacidad para testar del testador }\end{array}$ & Datos personales de los testigos \\
\hline Tipo de testamento & $\begin{array}{l}\text { Reconocimiento del notario de la } \\
\text { idoneidad de los testigos }\end{array}$ \\
\hline $\begin{array}{l}\text { Manifestación del testador de sus } \\
\text { datos personales }\end{array}$ & Datos personales del testador \\
\hline $\begin{array}{l}\text { Manifestación del testador de su } \\
\text { deseo de otorgar testamento }\end{array}$ & $\begin{array}{l}\text { Reconocimiento del notario de la } \\
\text { capacidad para testar del testador }\end{array}$ \\
\hline $\begin{array}{l}\text { Reconocimiento del notario de la } \\
\text { idoneidad de los testigos }\end{array}$ & $\begin{array}{l}\text { Manifestación del testador de su deseo } \\
\text { de otorgar testamento }\end{array}$ \\
\hline Cláusula de revocación & Cláusula de revocación \\
\hline $\begin{array}{l}\text { Reconocimiento del testamento y } \\
\text { conformidad del testador }\end{array}$ & Cláusulas del testamento \\
\hline Cláusulas del testamento & $\begin{array}{l}\text { Plasmación por escrito del cumplimiento } \\
\text { de las formalidades exigidas (lectura del } \\
\text { testamento, presencia de los testigos, } \\
\text { declaración de los testigos) }\end{array}$ \\
\hline Dación de fe del notario & Dación de fe del notario \\
\hline $\begin{array}{l}\text { Folios, serie y números en los que } \\
\text { se ha extendido el testamento }\end{array}$ & $\begin{array}{l}\text { Mención de las tachaduras y palabras } \\
\text { enmendadas presentes en el testamento }\end{array}$ \\
\hline $\begin{array}{l}\text { Firma del testador y de los testigos } \\
\text { en su caso }\end{array}$ & Fórmulas de cierre \\
\hline & Firma del testador y de los testigos \\
\hline
\end{tabular}

Como puede observarse, las macroestructuras referidas son muy similares. Esto se debe a que los ordenamientos jurídicos de los que se derivan tienen un origen común ${ }^{11}$, lo que hace que, en muchas ocasiones, la práctica jurídica se asemeje y ello se plasme en los diferentes documentos producidos. Ahora bien, esta proximidad puede actuar en ocasiones como un arma de doble filo para el traductor, que en ningún caso deberá conformarse con los equivalentes terminológicos propuestos por la mayoría de obras de consulta primarias $^{12}$ sin verificar previamente si dicha equivalencia existe también en el plano conceptual, para lo cual su búsqueda deberá ser más exhaustiva. Esto no sólo es aplicable desde el punto de vista de los equivalentes lingüísticos. Previamente, el traductor deberá analizar el texto (el testamento en este caso)

\footnotetext{
${ }^{11}$ El Derecho español y el Derecho francés son sistemas del denominado Derecho continental, que encuentra sus raíces en el Derecho romano y que se caracteriza, frente al denominado Derecho anglosajón, porque su principal fuente es la ley y porque sus normas están contenidas en cuerpos legales unitarios y ordenados (lo que solemos conocer con el nombre de códigos).

12 Nos referimos, fundamentalmente, a diccionarios bilingües generales y especializados.
} 
como un todo, un conjunto homogéneo en el que, como hemos señalado, el contenido y el continente no actúan de forma independiente sino que están íntimamente relacionados. En ese sentido, el conocimiento de las diferentes macroestructuras sirve para establecer los paralelismos pertinentes, pero el traductor debe abandonar la tendencia generalizada a sustituir la macroestructura existente en la LT por la macroestructura del texto en LO con el objeto de que el texto resultante, esto es, la traducción, se adecue más a la cultura hacia la cual se traduce ${ }^{13}$. Por lo general, en una traducción jurídica, por muy bien hecha que esté, se apreciará la labor del traductor, ya que los textos resultantes serán por lo general textos híbridos ${ }^{14}$. El traductor deberá discernir dónde está el límite en lo que al respeto del texto original se refiere y, en ese sentido, deberá combinar a la perfección la fidelidad hacia el contenido del texto en LO con la reexpresión de dicho contenido en LM, atendiendo para ello a la importancia y las características de los diferentes conceptos aquí abordados.

\section{LAS CONVENCIONES TEXTUALES}

El testamento, al igual que otros muchos documentos, de origen jurídico o no, responde a determinadas convenciones textuales. Isabel Cómitre (2006: 122) se refiere a este concepto como

"un marco que determina ciertas características comunes
de los textos: cómo se construyen, estructuran y qué
características lingüísticas y estilísticas los componen y
singularizan frente a otros textos."

A nuestro parecer, esta definición abarca el concepto de macroestructura tal y como anteriormente lo hemos entendido e incluye, además, otros aspectos que pertenecerían a un nivel inferior como son las características lingüísticas y estilísticas de los tipos de textos.

\footnotetext{
13 A este respecto, las palabras de Anabel Borja (2007b: 45) nos parecen ser muy precisas cuando afirma que "el objetivo principal de la traducción es generar un texto en lengua meta que, sin pretender crear la ilusión de ser un documento original, salvaguarde la función jurídica del texto original utilizando un estilo y un registro jurídico apropiados y respetuosos con las convenciones de género. No se trata de reproducir elementos lingǘsticos del original, sino de encontrar recursos para mantener la identidad expresiva entre el original y la traducción."

${ }^{14}$ Retomamos la idea expresada por Didier (1990), según el cual "De la confrontation des textes sortira un tertium quid, une autre langue, un hybride en somme qui n'est ni tout à fait la copie de l'original ni vraiment un texte conforme, dans la lettre comme dans l'esprit, au système d'arrivée."
} 
En este nivel, el traductor ya debe considerar aquellos factores mediante los cuales se actualiza el contenido de un documento. Entre ellos, destacamos el uso del lenguaje jurídico, de la gramática general, de determinadas convenciones ortotipográficas, así como las tendencias estilísticas propias del tipo de texto en cuestión. La consideración de todos estos aspectos resulta primordial en el traslado de la información de una lengua a otra. En cuanto al elemento estilístico, Emilio Ortega (1997: 140) señala que "es fundamental para no ser traidores al continente del texto original. [...] con el componente estilístico tratamos la forma en que el contenido se presenta."

El lenguaje del testament authentique posee algunas de las características generales que tradicionalmente se le atribuyen al lenguaje jurídico general. Así, en lo que al nivel léxico se refiere, hace gala de un vocabulario especializado propio del ámbito del cual el documento procede. Es lo que, en el seno de las denominadas lenguas de especialidad, se conoce como terminología. Dentro del nivel sintáctico, es frecuente el uso de oraciones relativamente largas que responden a una fraseología propia cuyo conocimiento y manejo tanto en la LO como en la LT contribuirán a realizar una traducción satisfactoria. Dicha fraseología no sólo responde a un determinado uso estilístico del lenguaje sino que, además, sirve para vehicular la carga jurídica del documento. El traductor deberá ser especialmente cauteloso a la hora de trasladar el contenido así expresado. Uno de los mayores obstáculos que encontrará será la escasa información fraseológica que contienen las obras de consulta. Por ello, abogamos por la realización de estudios en este sentido, estudios basados en corpus delimitados con el objeto de analizarlos teniendo en cuenta parámetros como la ubicación de los textos, sus macroestructuras y sus características lingǘsticas. Para ilustrar lo que acabamos de exponer, retomamos a continuación la fórmula de cierre que suele aparecer en todos los testaments authentiques:

"Et, après lecture faite en entier des présentes par le notaire soussigné, le testateur a signé avec les témoins et ledit notaire, le tout en la présence réelle, simultanée et non interrompue des témoins et du notaire."

En España, la fórmula correspondiente suele expresarse como sigue ${ }^{15}$ :

"Leído por mí el notario íntegramente y en alta voz este testamento al testador por renunciar al derecho que le advierto

15 Esta fórmula puede presentar pequeñas variaciones pero, en esencia, el contenido es siempre el mismo. 
tiene de hacerlo por sí, encontrándolo conforme presta su consentimiento y firma."

La tendencia del traductor podría tal vez ser el sustituir una fórmula por otra. Sin embargo, dicha elección no se puede realizar arbitrariamente sin considerar que la información contenida en la fórmula española no recoge toda la información de la fórmula francesa ${ }^{16}$. En este punto, el traductor deberá preguntarse hasta qué punto dicha diferencia de contenido podría tener repercusión en la validez del documento. A este respecto, nuestra posición es clara: la traducción profesional de un testamento (testament authentique francés en este caso) no se entiende si dicho documento no está inmerso en un proceso de repartición de herencia ${ }^{17}$. En este caso, el ordenamiento jurídico que rige el testamento francés será el ordenamiento francés, por lo que se habrá de respetar al máximo su contenido, empleando, eso sí, los medios lingüísticos adecuados en nuestra lengua e intentando adecuar la traducción a las convenciones de la cultura de la LM en la medida de lo posible.

Por último, nos gustaría referirnos al uso específico de determinadas convenciones ortotipográficas dentro del lenguaje empleado en los testamentos franceses, concretamente, al uso de las mayúsculas.

Según Xosé Castro y Lucía Rodríguez (1999), la ortotipografía se puede definir como "la materia que trata la correcta acentuación y puntuación de los textos, además de la correcta utilización de ciertos signos complementarios".

En lo que al uso de las mayúsculas se refiere, los mismos autores afirman que se suelen utilizar en títulos de libros, cabeceras de publicaciones, siglas y en los verbos de contratos y documentos jurídicos o administrativos (EXPONE, SOLICITA, COMPARECE).

En efecto, en el testament authentique encontramos este uso de las mayúsculas. El ejemplo más característico es la expresión $A C O M P A R U$ (COMPARECE). Pero las mayúsculas también aparecen en otras partes del testamento, siempre con un claro valor demarcativo. Así, las expresiones $P A R$ DEV ANT, LEQUEL y DONT ACTE ${ }^{18}$, situadas al comienzo del documento, tras los datos personales del compareciente y como fórmula de cierre respectivamente, dan fe de ello. La expresión de la fecha, situada justo al comienzo del documento, también suele ir en mayúsculas.

\footnotetext{
${ }^{16} \mathrm{El}$ ordenamiento jurídico francés exige que se haga referencia a la presencia de los testigos. El ordenamiento jurídico español, por su parte, obvia este aspecto pero sí alude al derecho que el testador tiene de leer el testamento por sí mismo.

${ }^{17}$ En cuyo caso, además, la traducción requerida habrá de ser una traducción jurada, realizada únicamente por aquellos profesionales debidamente habilitados.

${ }_{18}$ Las tres expresiones forman parte de fórmulas fraseológicas más extensas propias de los testamentos: "PAR DEVANT Maitre..., notaire à..., soussigné"; "LEQUEL, sain d'esprit ainsi qu'il est apparu au notaire et aux témoins..."; "DONT ACTE, rédigé sur...pages".
} 
En un testamento abierto español, este uso coincide en la expresión de la fecha y el lugar de otorgamiento y en el verbo COMPARECE. Otras fórmulas como Ante mi o Doy fe suelen aparecer en minúscula.

No se trata aquí del respeto de la normativa de obligado cumplimiento en cuanto a las convenciones ortotipográficas establecidas por los organismos encargados de cada comunidad lingüística, sino de un uso determinado de algunos signos que, si bien respetan la gramática y la ortografía de la lengua en la que se insertan, suponen un uso específico de algunos elementos con un objetivo claro. El traductor deberá ser conocedor de ello e intentar siempre mantener un cierto equilibrio entre las lenguas de origen y de llegada.

\section{CONCLUSIÓN}

Como hemos visto, el proceso traductor debe poner en marcha toda una serie de mecanismos con respecto al análisis del texto original. Para nosotros, es imprescindible la consideración y el respeto de las fases de pretraducción antes de acometer el proceso traslativo propiamente dicho y, en ese sentido, los conceptos de género, macroestructura y convenciones textuales aquí retomados y aplicados al caso concreto de un tipo de documento jurídico dado, cobran una especial relevancia y pueden ayudar no sólo a la traducción de un documento único sino que, además, pueden derivar en la sistematización y clasificación de las características propias de un tipo de texto con vistas a la traducción.

\section{BIBLIOGRAFÍA}

Álvarez de Morales, A. y M.A. Del Arco Torres (2001): Formularios de herencias. Granada, Comares.

Borja Albi, Anabel (2007A): "Los géneros jurídicos" en Alcaraz Varó, E., Mateo Martínez. J. y Yus Ramos, F. (eds): Las lenguas profesionales y académicas. Barcelona, Ariel Lenguas Modernas.

BORJA Albi, ANABEL (2007B): Estrategias, materiales y recursos para la traducción jurídica inglés-español. Castellón de la Plana, Publicacions de la Universitat Jaume I.

Campos Pardillo, Miguel Ángel (2007): "El lenguaje de las ciencias jurídicas. Nuevos retos y nuevas visiones" en Alcaraz Varó, E., Mateo Martínez. J. Y Yus Ramos, F. (eds): Las lenguas profesionales y académicas. Barcelona, Ariel Lenguas Modernas. 
CAstro RoIG, XOsÉ. Y RODRÍGUEZ CORRAL, LuCÍA (1999): Errores ortotipográficos en la traducción al español [en línea]: http://www.xcastro.com/ortotipo.html. [Consulta: 01/02/2009].

CÓMITRE NARVÁEZ, ISABEL (2006): "Las convenciones estilísticas culturales en campañas publicitarias de promoción turística: el caso de 'España marca' (Turespaña)" en Féliz Fernández, L. y Mata Pastor, C. (eds): Traducción y cultura. Málaga, Encasa.

GARCÍA IZQUIERDO, ISABEL (2007): "Los géneros y las lenguas de especialidad" en Alcaraz Varó, E., Mateo Martínez. J. Y Yus Ramos, F. (eds): Las lenguas profesionales y académicas. Barcelona, Ariel Lenguas Modernas.

Hurtado Albir, AMPARO (1999): Enseñar a traducir. Madrid, Edelsa.

Ortega ArJonilla, EMILIO (1997): "El proceso de traducción de documentos jurídicos” en San Ginés Aguilar, P. Y Ortega Arjonilla, E. (eds): Introducción a la traducción jurídica y jurada (francés-español). Granada, Comares.

VALDERREY REÑONES, CRISTINA (2006): "Convenciones textuales y estrategia traslativa” en Féliz Fernández, L. Y Mata Pastor, C. (eds): Traducción y cultura. Málaga, Encasa.

VAN DiJK, TEUN (1980): Estructuras y funciones del discurso. Madrid, Siglo XXI.

VÁZQUEZ Y DEL ÁRBOL, EsTHER (2008): La traducción (inglés-español) de testamentos británicos y documentos relacionados. De la teoría a la práctica. Granada, Universidad de Granada. 